\title{
Cocaine Hydrochloride
}

National Cancer Institute

\section{Source}

National Cancer Institute. Cocaine Hydrochloride. NCI Thesaurus. Code C81016.

The hydrochloride salt form of the tropane alkaloid cocaine, with central nervous systems (CNS) stimulating and local anesthetic activity. Cocaine binds to the dopamine, serotonin, and norepinephrine transport proteins and inhibits the re-uptake of dopamine, serotonin, and norepinephrine into pre-synaptic neurons. This leads to an accumulation of the respective neurotransmitters in the synaptic cleft and may result in increased postsynaptic receptor activation. Its effect on dopamine levels causes CNS stimulation and euphoria, and ultimately dependence. The mechanism of action through which cocaine exerts its local anesthetic effects is by binding to and blocking the voltage-gated sodium channels in the neuronal cell membrane. By stabilizing neuronal membranes, cocaine inhibits the initiation and conduction of nerve impulses and produces a reversible loss of sensation. 